\title{
Evaluación del uso de acetileno como fuente de carbono en la carburización de aceros
}

\section{Evaluation of using acetylene as carbon source in the carburization of steels}

\author{
Claudia Patricia Serna Giraldo ${ }^{1}$, Oscar Eduardo Rios Diez ${ }^{1}$, \\ Rufino Medina Aguas ${ }^{1}$, Maria Camila Lasso Delgado ${ }^{1}$, \\ Ricardo Emilio Aristizabal Sierra ${ }^{1}$
}

\footnotetext{
${ }^{1}$ Grupo de Investigaciones Pirometalúrgicas y de materiales - GIPIMME- Departamento de Ingeniería de Materiales, Facultad de Ingeniería, Universidad de Antioquia, Calle 67 Número 53- 108, Medellín, Medellín, Colombia. e-mail: claudia.serna@udea.edu.co; eduardo.rios@udea.edu.co; rufinomedina@udea.edu.co; mariacamila.lasso@hotmail.com; ricardo.aristizabal@udea.edu.co
}

\section{RESUMEN}

En este trabajo fue evaluado el efecto del uso de acetileno como fuente de carbono en la carburización gaseosa del acero AISI/SAE 8620. Para esto se diseñó y fabricó un sistema de carburización gaseosa con las siguientes características: producción controlada de una atmósfera gaseosa carburante, zona de reacción isotérmica inerte y hermética y sonda de carbono/oxígeno para el control del potencial de carbono (Cp) durante el ciclo de carburización. Se utilizó una atmósfera compuesta por un gas endotérmico sintético de composición $40 \% \mathrm{H}_{2}, 40 \% \mathrm{~N}_{2}$ y $20 \% \mathrm{CO}$ con flujo constante de $100 \mathrm{~cm}^{3} / \mathrm{min}$ y variaciones de acetileno con flujos de $40 \mathrm{~cm}^{3} / \mathrm{min}, 65 \mathrm{~cm}^{3} / \mathrm{min}$ y $85 \mathrm{~cm}^{3} / \mathrm{min}$. Estos gases se inyectaron en el reactor a $900^{\circ} \mathrm{C}$ y se sostuvieron por tres horas. Se seleccionó gas acetileno como agente enriquecedor de carbono en la atmósfera debido a los elevados ratios de descomposición que presentan estas moléculas y a la baja tendencia a desarrollar reacciones de polimerización. La caracterización de los aceros carburizados se realizó mediante microscopia óptica (MO), espectrometría de emisión óptica (EEO) y ensayos de microdureza Vickers (HV). Los resultados revelan que existe una gran eficiencia y control en la transferencia de carbono durante el ciclo de carburización, favoreciendo la optimización de recursos y tiempos de proceso.

Palabras clave: carburización gaseosa, acetileno, gas endotérmico sintético, aceros bajo carbono.

\begin{abstract}
The effect of using acetylene as a carbon source in the gas carburization of AISI/SAE 8620 steel was evaluated in this work. For this purpose, a gaseous carburizing system was designed and manufactured with the following characteristics: controlled production of a carburant gaseous atmosphere, inert and hermetic isothermal reaction zone and carbon/oxygen probe for the control of carbon potential $(\mathrm{Cp})$ during the carburization cycle. An atmosphere composed of synthetic endothermic gas with composition of $40 \% \mathrm{H}_{2}, 40 \% \mathrm{~N}_{2}$ and $20 \% \mathrm{CO}$ and constant flow of $100 \mathrm{~cm}^{3} / \mathrm{min}$ and acetylene variations with flows of $40 \mathrm{~cm}^{3} / \mathrm{min}, 65 \mathrm{~cm}^{3} / \mathrm{min}$ and $85 \mathrm{~cm}^{3} / \mathrm{min}$ was used. These gases were injected into the reactor at $900^{\circ} \mathrm{C}$ and held for three hours. The acetylene gas was selected as the carbon-enriching agent in the atmosphere, due to the high decomposition ratios of these molecules and the low tendency to develop polymerization reactions. The characterization of the carburized steels was performed by optical microscopy (OM), optical emission spectrometry (OES) and Vickers microhardness tests (HV). The results show that there is a great efficiency and control in the carbon transfer during the carburization cycle, favoring the optimization of resources and processing times.
\end{abstract}

Keywords: gas carburizing, acetylene, synthetic endothermic gas, low carbon steels.

\section{INTRODUCCIÓN}

La carburización es un tratamiento térmico por el cual el contenido de carbono de la superficie en una pieza de acero con bajo carbono aumenta debido a la exposición a una atmósfera rica en carbono a una temperatura 
dentro del campo de fase austenítico [1]. La introducción del carbono en la austenita durante la carburización está influenciada por dos procesos importantes: uno es la reacción que causa que el carbono sea absorbido en la superficie del acero; y el otro es la velocidad a la cual el carbono puede difundirse desde la superficie hacia el interior del acero [2, 3]. El carbono se introduce por el uso de atmósferas gaseosas (cementación gaseosa), baño de sales (cementación líquida) y compuestos sólidos (cementación en caja). En los procesos de carburización con agentes carburantes sólidos o líquidos, se obtiene el gas carburante en la proximidad inmediata de la superficie que se va a carburar. El gas formado en estas condiciones tiene una composición química apenas controlable y solo se puede modificar limitadamente, por esta razón se ha difundido la utilización de la carburización gaseosa la cual además, brinda capas carburadas más gruesas que las dadas por los otros métodos entre otras razones porque: la temperatura de carburización se puede escoger entre límites amplios, es posible ajustar la cantidad de gas carburante al área de la superficie que se va a carburar, puede variarse la actividad del carburante y es posible acelerar la carburización por adición de gases carburantes [2].

Las atmósferas de carburización gaseosa consisten en una mezcla de agentes carburantes como $\mathrm{CO}$, $\mathrm{CH}_{4}, \mathrm{C}_{2} \mathrm{H}_{2}$, entre otros, y agentes descarburantes como $\mathrm{CO}_{2}$ y $\mathrm{H}_{2} \mathrm{O}$, cuya relación determina el potencial de carburización en el horno [4]. Como la fuerza motriz para la carburización está determinada por el potencial de carbono, es necesario mantener un alto potencial de carburización durante todo el proceso. Desde el punto de vista termodinámico, la generación de la atmósfera carburante es un proceso bastante complejo que implica la interacción de numerosos gases. El gas portador que entra al horno está compuesto generalmente por $\mathrm{CO}, \mathrm{CO}_{2}, \mathrm{CH}_{4}, \mathrm{C}_{2} \mathrm{H}_{2}, \mathrm{H}_{2}, \mathrm{H}_{2} \mathrm{O}$ y $\mathrm{N}_{2}$ [5]. Se ha estimado que aproximadamente 180 reacciones químicas se producen simultáneamente en la atmósfera de carburización, entre las cuales solo son importantes las reacciones descritas por las ecuaciones 1,2 y $3[3,5,6]$, las cuales determinan la velocidad de transferencia de carbono desde la atmósfera hasta la superficie del acero.

$$
\begin{aligned}
2 \mathrm{CO} & \leftrightarrow \mathrm{C}(\gamma)+\mathrm{CO}_{2} \\
\mathrm{CO}+\mathrm{H}_{2} & \leftrightarrow \mathrm{C}(\gamma)+\mathrm{H}_{2} \mathrm{O} \\
\mathrm{CO}_{2}+\mathrm{H}_{2} & \leftrightarrow 2 \mathrm{C}(\gamma)+\mathrm{H}_{2} \mathrm{O}
\end{aligned}
$$

Mientras la carburización se produce más rápidamente por la descomposición del $\mathrm{CO}$, los subproductos de las reacciones de carburización, $\mathrm{CO}_{2}$ y $\mathrm{H}_{2} \mathrm{O}$, actúan como agentes descarburantes. La presencia de $\mathrm{CO}_{2}$ requiere una elevada concentración de $\mathrm{CO}$ para equilibrar esta acción. Por lo tanto, para que el proceso continúe, estas especies descarburantes deben ser reducidas. Por esto el gas endotérmico se enriquece mezclándolo con un gas hidrocarbonáceo [5]. El propósito de este gas es reaccionar con el $\mathrm{CO}_{2}$ y el $\mathrm{H}_{2} \mathrm{O}$, reduciendo así su concentración y produciendo más carbono naciente, $\mathrm{CO}$ y $\mathrm{H}_{2}$ como productos de reacción, como se evidencia en la reacción descrita por la ecuación 4 [7], la cual ilustra la descomposición de la molécula de acetileno.

$$
\mathrm{C}_{2} \mathrm{H}_{2} \leftrightarrow 2 \mathrm{C}(\gamma)+\mathrm{H}_{2}
$$

En la carburización en medio gaseoso las reacciones de descomposición de las moléculas del hidrocarburo tienen lugar por descomposición térmica o pirolisis [8]. Cuando se utiliza acetileno, el hidrocarburo se mezcla con nitrógeno, el cual actúa como diluyente, además, de disminuir la actividad del gas principal o para reducir la tendencia a la formación de residuos sólidos carbonosos [7]. Tanto el nitrógeno como el hidrógeno, actúan también como gas de transporte del acetileno durante el proceso.

Se han conseguido mejores resultados utilizando gases hidrocarburos no saturados, tales como el eteno y particularmente acetileno. La razón consiste en las elevadas velocidades de descomposición que presentan estas moléculas en las condiciones de carburización en atmósferas gaseosas, así como a la baja tendencia a desarrollar reacciones de polimerización [5]. La molécula de acetileno se descompone en varias etapas intermedias, como se aprecia en la figura 1 , si bien en general pueden resumirse en la formación de $\mathrm{C}, \mathrm{H}_{2}$ y pequeñas cantidades de $\mathrm{CH}_{4}[9]$. 


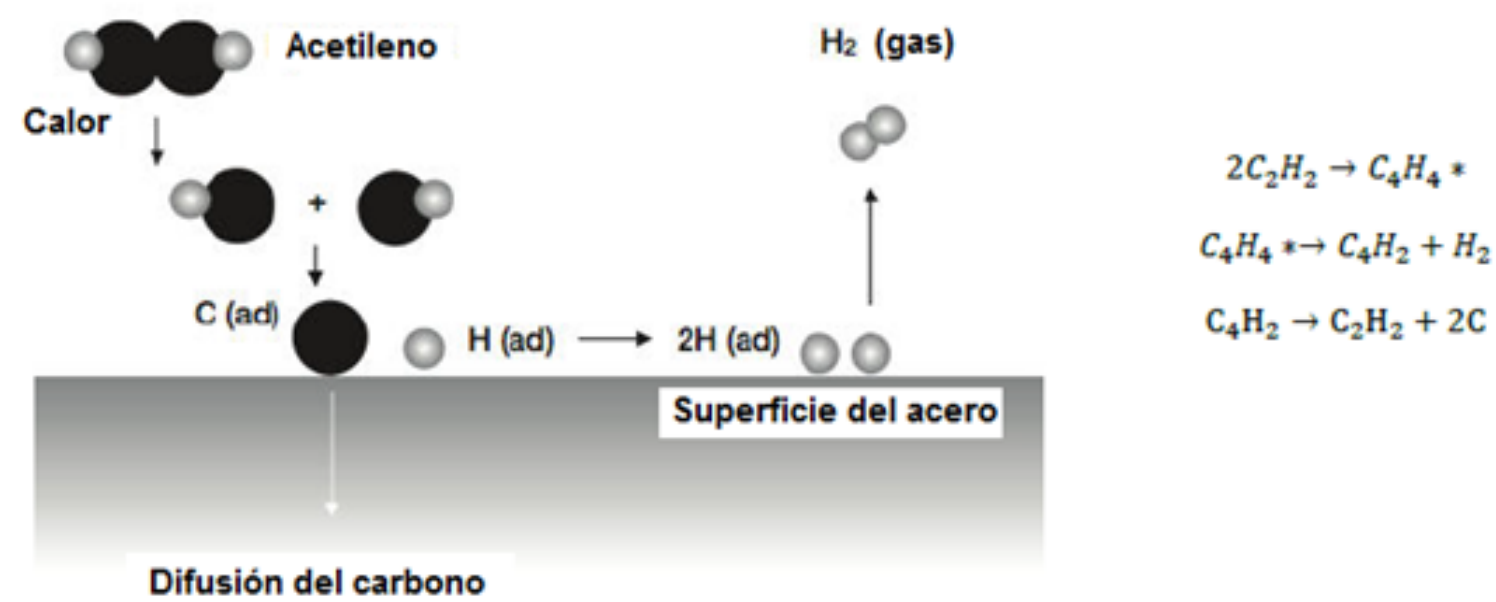

Figura 1: Mecanismo de disociación del acetileno en la superficie del acero [7].

La gran aceptación que tiene el acetileno como medio de carburización, se basa en que este gas tiende a disociarse solo en la superficie del acero, y por lo tanto se consigue que penetre de forma eficaz incluso en componentes profundos y de geometrías complejas [10]. Por otra parte el acetileno en su descomposición se disocia en dos átomos de carbono activos sin formar fragmentos vinílicos (en comparación con el único átomo de carbono más los fragmentos de moléculas secundarias producidas por la disociación del propano y el etileno) [3].

En sistemas de atmósferas carburantes el uso del acetileno está restringido al uso en sistemas de vacío, mientras que los sistemas que operan a presiones mayores a 100mbar [9] (o presión atmosférica) se caracterizan por la utilización de gases enriquecedores de carbono como el propano, etano y el etileno [11]. Los inconvenientes ligados a la utilización de estos gases es que las reacciones de descomposición térmica dan lugar a productos residuales formados por estructuras de hidrocarburos de cadena larga, que se presentan en forma de hollín que progresivamente se depositan sobre los elementos internos de los hornos y las instalaciones de carburización, lo que provoca una disminución en la vida útil del equipamiento, así como la reducción de la eficiencia del proceso de carburización [3], [7]. Es por esto que el presente trabajo se presenta la utilización de acetileno como fuente de carbono en la carburización gaseosa del acero AISI8620, bajo condiciones de carburización atmosférica; a fin de evidenciar qué influencia tiene la adición de acetileno en las reacciones durante el proceso.

\section{MATERIALES Y MÉTODOS}

Se utilizaron probetas cilíndricas de acero AISI/SAE 8620 comercial de 50mm de diámetro cuya composición química se determinó mediante espectrometría de emisión óptica (EEO) en un equipo marca Bruker Magellan modelo Q8. Para los tratamientos térmicos de carburización gaseosa se utilizó el sistema presentado en la figura 2, el cual consta de una zona de preparación de la atmósfera o de mezcla de gases a utilizar, zona de reacción o carburización y sistema de control de atmósfera mediante una sonda de carbono/oxígeno. Para la carburización se utilizó gas endotérmico sintético de composición $20 \% \mathrm{CO}, 40 \% \mathrm{H}_{2}$ y $40 \% \mathrm{~N}_{2}$, el cual se fijó en una tasa de $100 \mathrm{~cm}^{3} / \mathrm{min}$ y al que se le adicionó gas acetileno con flujos de $85 \mathrm{~cm}^{3} / \mathrm{min}, 65 \mathrm{~cm}^{3} / \mathrm{min}$ y $40 \mathrm{~cm}^{3} / \mathrm{min}$, con el objetivo de evaluar la eficiencia en la transferencia de carbono durante el ciclo de carburización. En la tabla 1 se aprecian con más detalle las condiciones de ensayo.

El reactor que está fabricado en acero al carbono, tiene la capacidad de trabajar tanto de forma discontinua, como un reactor tipo Batch, o de forma continua como un reactor de tanque agitado (RCTA) o flujo pistón (PFR). Para evaluar el efecto del acetileno en la carburización del acero AISI/SAE 8620, se eligió la condición PFR, donde la concentración del gas carburante se varió continuamente en la dirección longitudinal, pero no en la dirección transversal al flujo. Para definir los volúmenes de trabajo se realizaron simulaciones del comportamiento de las especies que participan en el proceso de carburación según las reacciones representadas en las ecuaciones $1,2,3$ y $4[6,9,10,11]$, utilizando el programa Polymath $6.0^{\circledR}$. Además, se calcularon la concentración de las especies antes y después de las reacciones, así como la velocidad de la reacción, las cuales dependen del flujo de los gases que se utilizan para el proceso de carburización. 


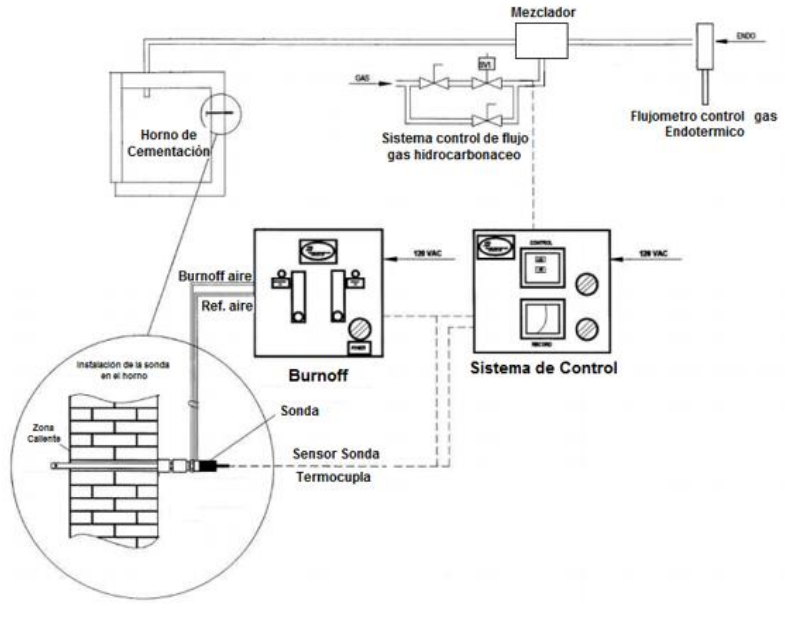

(a)

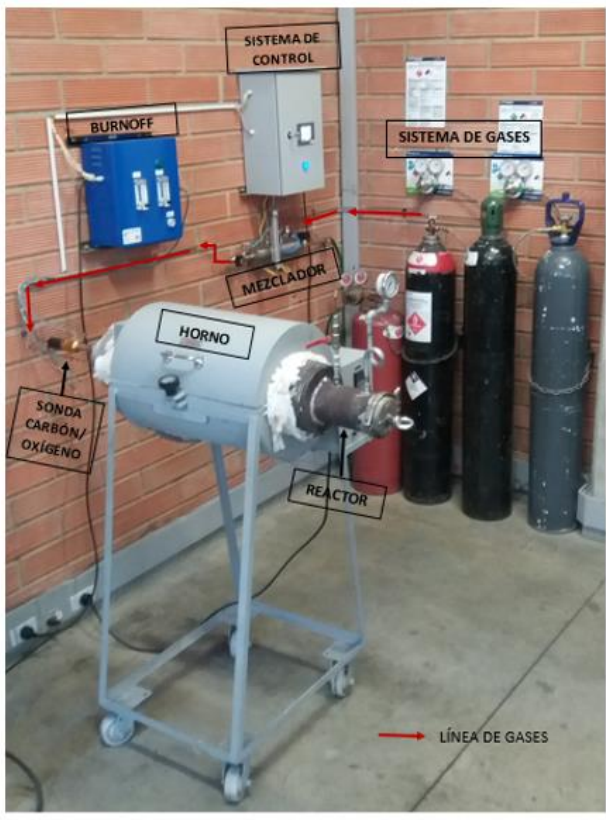

(b)

Figura 2: Equipo de carburización gaseosa. Grupo GIPIMME - Universidad de Antioquia: (a) esquema del sistema de carburización, (b) equipo de carburización gaseosa.

Tabla 1: Factores y Niveles del diseño de experimentos.

\begin{tabular}{c|c|c|c|c}
\hline MUESTRA & NIVEL & FLUJO DE ACETILENO $\left(\mathbf{c m}^{\mathbf{3}} / \mathbf{m i n}\right)$ & TEMPERATURA $\left({ }^{\circ} \mathbf{C}\right)$ & TIEMPO (horas) \\
\hline M1 & Bajo & 40 & & \\
M2 & Medio & 65 & \multirow{2}{*}{900} & 3 \\
\cline { 1 - 2 } M3 & Alto & 85 & & \\
\hline
\end{tabular}

En todas las probetas se realizaron medidas de microdureza Vickers posterior a cada tratamiento térmico. Este ensayo se llevó a cabo de acuerdo a las norma ASTM E92-82 [15]. Se utilizó un microdurómetro Shimadzu HMV-G 20DT, con cargas de 100g-f, y 15 segundos de sostenimiento y se hizo un barrido desde la superficie hasta el núcleo cada $50 \mu \mathrm{m}$, se realizaron 8 indentaciones por muestra, en un total de tres probetas por ensayo.

La caracterización microestructural, se realizó mediante microscopía óptica (MO) en un microscopio marca Nikon modelo MA 100. Las secciones de las probetas se prepararon con lijas de tamaño de grano número ASTM 120, 240, 320, 400, 600, 1000, seguido de pulido en paño con partículas de alúmina de $3 \mu \mathrm{m}$ y $1 \mu \mathrm{m}$, de acuerdo a la norma de preparación de muestras ASTM E3 [16]. El ataque químico se llevó a cabo usando reactivo Nital $2 \%$.

\section{RESULTADOS Y DISCUSIÓN}

Los resultados de la composición química del acero AISI/SAE 8620, obtenida mediante espectrometría de emisión óptica se observan en la tabla 2. En el caso del AISI/SAE 8620 elementos como el Cr, Ni, Mo aceleran la precipitación de carburos, que solo disolverán a altas temperaturas. También presentan una fuerte influencia en la difusión del carbono y en consecuencia pueden reducir la profundidad de la capa carburada [10].

En la figura 3 se puede observar la microestructura en estado de entrega del acero AISI/SAE 8620. Este presenta una estructura ferrítico/perlítica, en la cual la ferrita es la fase clara y la perlita el constituyente oscuro, con número de grano 6.5 que corresponde a un tamaño de grano medio de $38 \mu \mathrm{m}$. 
Tabla 2: Composición química del acero AISI/SAE $8620, \%$ en peso.

\begin{tabular}{l|l}
\hline ELEMENTO $(\% \mathrm{~W})$ & ACERO \\
\hline $\mathrm{C}$ & 0.213 \\
\hline $\mathrm{Si}$ & 0.254 \\
\hline $\mathrm{Mn}$ & 0.712 \\
\hline $\mathrm{Cr}$ & 0.456 \\
\hline $\mathrm{Ni}$ & 0.471 \\
\hline $\mathrm{P}$ & 0.025 \\
\hline $\mathrm{S}$ & 0.019 \\
\hline $\mathrm{Mo}$ & 0.185 \\
\hline
\end{tabular}

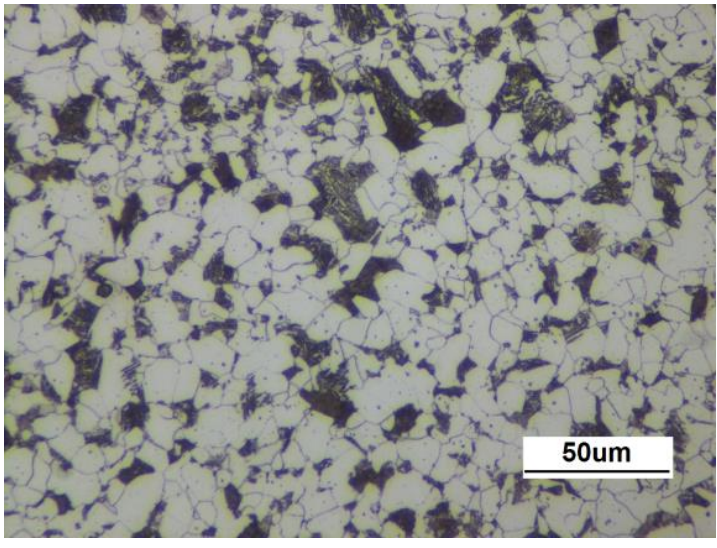

Figura 3: Micrografía acero AISI/SAE 8620 en estado de entrega. Sección transversal, ataque con $2 \%$ Nital.

En el sistema empleado, la atmósfera se caracteriza por las altas concentraciones de elementos y gases reactivos, los cuales garantizan que la velocidad y cantidad de carbono transferido a la superficie del acero sea controlada a partir de la composición de la atmósfera. En la figura 4 se presentan los resultados arrojados por la simulación de la concentración de los gases, antes del proceso de carburización y después de las reacciones en el interior del reactor, se aprecia la concentración de las especies que se consumen y se producen dentro del reactor a la temperatura de austenización después que los gases endotérmico y acetileno son inyectados.

De la figura 4 se observa que la velocidad de descomposición del acetileno es mayor cuando el flujo de este es mayor, favoreciendo una mayor generación de carbono libre, disponible para la carburización, como se logra observar en la ecuación 4, acompañada de una mayor cantidad de $\mathrm{H}_{2}$. Las reacciones de descomposición de las moléculas de acetileno tienen lugar por descomposición térmica o de pirólisis, la cual a la temperatura en que se realizó el proceso de carburización, se disocia siguiendo reacciones complejas, y en su última etapa requiere una superficie metálica que actúa como catalizador. A fin de minimizar la formación de largas cadenas de hidrocarburo, se introducen altas concentraciones de nitrógeno e hidrógeno en el gas endotérmico sintético durante el proceso de carburización. La introducción del $40 \%$ de nitrógeno respecto al caudal total resulta en una reducción de las reacciones de aromatización. Por otra parte, el efecto del hidrógeno consiste en limitar las reacciones de deshidrogenación. De otro lado, en la figura 4 se observa que la cantidad de $\mathrm{CO}$ permanece aproximadamente constante, al igual que la cantidad de $\mathrm{CO}_{2} \mathrm{y}_{2} \mathrm{O}$, las cuales son prácticamente nulas. Estas especies se producen de acuerdo con las ecuaciones de reacción 1, 2 y 3, las cuales se favorecen en el sentido de los reactivos. Una limitación ligada al uso de gases enriquecedores de atmósfera, como en el caso del acetileno, es que las reacciones de descomposición térmica dan lugar a productos residuales formados por estructuras de hidrocarburos de cadena larga, que se presentan en forma de hollín los cuales progresivamente se depositan sobre el interior del horno. Los factores responsables de la formación de estos compuestos son el elevado tiempo de residencia, la temperatura, y la generación de gran cantidad de $\mathrm{CO}_{2} \mathrm{y} \mathrm{H}_{2} \mathrm{O}$. 


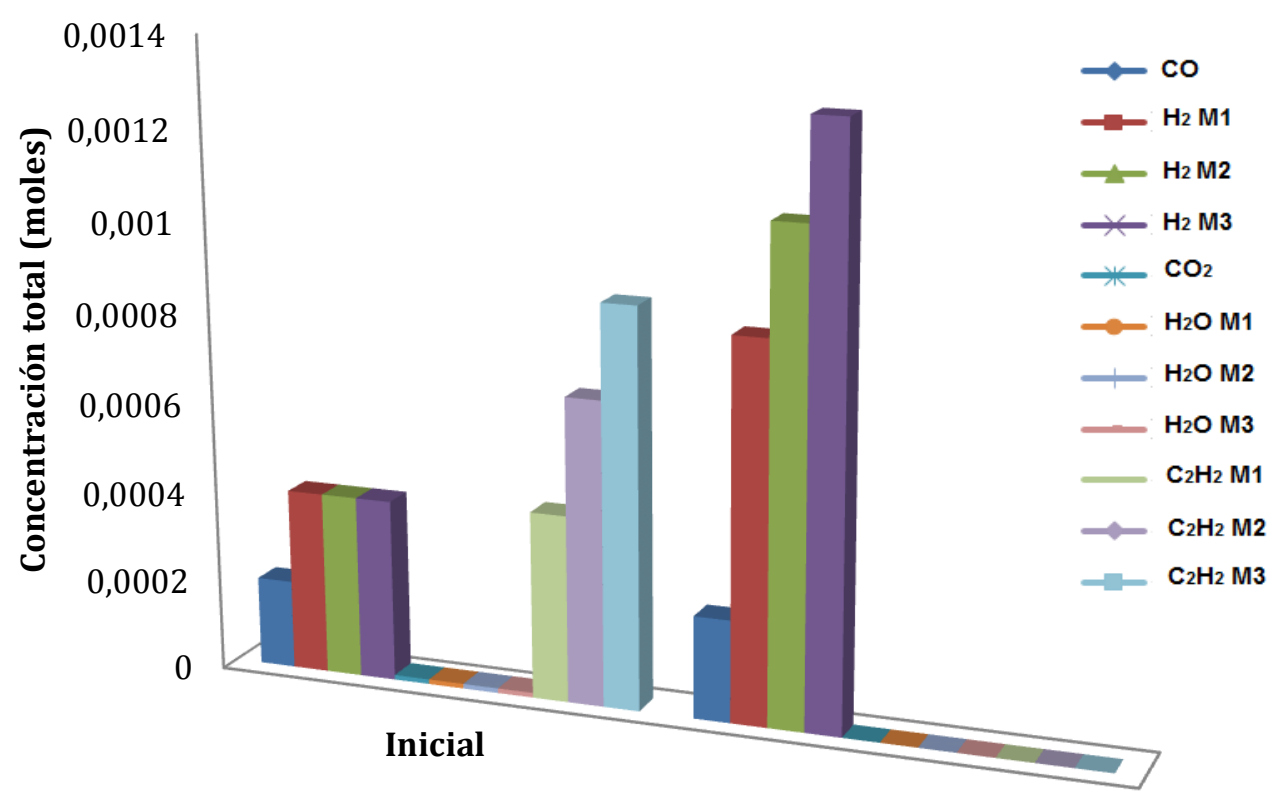

Despues de Reacciones

Figura 4: Variación en concentración de moles de las especies participantes del proceso de carburización gaseosa mediante el uso de gas acetileno como agente enriquecedor de atmósfera carburante.

En la tabla 3 se muestra la profundidad alcanzada en cada una de las capas carburadas, así como el contenido máximo de carbono alcanzado en superficie, y medido en la atmósfera por la sonda de carbono/oxígeno. Se observa que en la medida que el flujo de acetileno se aumenta, la concentración de carbono en la superficie de la pieza aumenta, además de la profundidad de la capa carburizada. Como la temperatura y el tiempo de carburización se mantuvieron constantes en las tres muestras, este aumento se explica por el aumento de carbono disponible en la atmósfera, lo cual aumenta la velocidad de difusión del carbono al aumentar el gradiente de concentración y por ende la fuerza impulsora para la difusión hacia el interior del acero.

Tabla 3: Profundidad de capa carburada y contenido máximo de carbono en superficie para las muestras ensayadas.

\begin{tabular}{c|c|c|c|c}
\hline \multirow{2}{*}{ Muestra } & $\begin{array}{c}\text { Flujo de acetileno } \\
\left(\mathbf{c m}^{\mathbf{3}} / \mathbf{m i n}\right)\end{array}$ & $\begin{array}{c}\text { Profundidad de capa } \\
\text { carburada }(\boldsymbol{\mu m})\end{array}$ & $\begin{array}{c}\text { Composición en } \\
\text { la superficie } \\
(\boldsymbol{\%} \mathbf{C})\end{array}$ & $\begin{array}{c}\text { Carbono medido por } \\
\text { sonda carbono/oxígeno } \\
(\boldsymbol{\%})\end{array}$ \\
\hline M1 & 40 & 150 & 0.5 & 0.55 \\
\hline M2 & 65 & 350 & 0.8 & 0.86 \\
\hline M3 & 85 & 450 & 1.1 & 1.18 \\
\hline
\end{tabular}

En la figura 5 se aprecian los perfiles de concentración de carbono y microdureza Vickers a lo largo de la capa carburada para cada una de las muestras evaluadas, M1, M2 y M3. Con respecto al porcentaje de carbono, en las figura 5a, 5c y 5e se aprecian los perfiles que indican la concentración en carbono a lo largo de la capa carburada, el enriquecimiento está basado en el potencial de carbono de la atmósfera carburante.

La cantidad de carbono que es absorbido por la superficie del acero depende de la temperatura, el caudal de hidrocarburo, la superficie de las piezas y la presión parcial de la mezcla gaseosa empleada durante el proceso [13]. Como en este caso la única variable es el flujo de acetileno, se observa que el rendimiento es significativamente mayor cuando se presenta un mayor flujo de acetileno lo cual se evidencia para la muestra M3, en la figura 5e; esto debido a que la transferencia de carbono desde la atmósfera hacia la superficie del metal es lineal en el dominio situado por debajo de la saturación de la austenita. Cuando el caudal es mayor que la superficie crítica (relación entre el área de las piezas y el flujo de gas carburante), el flujo de carbono 
aumenta linealmente con el caudal de gas, llegando a un punto en el cual el flujo se estabiliza aunque el caudal siga aumentando, favoreciendo la formación de hollín.

Se destaca que existe un desajuste en el perfil de concentración de carbono teórico y experimental a lo largo de la capa carburizada, especialmente para la muestra M1, figura 5a. Este comportamiento en parte se puede explicar por las condiciones de operación, como son el sistema de carburización y la pureza de los gases usados, la cual puede afectar la cantidad de carbono disponible por unidad de volumen. Pero el aspecto que más influencia puede tener, principalmente en la condición de bajo flujo, como en el caso de la muestra M1, es el caudal másico de gas de carburización disponible, debido a que la dosificación de gas por debajo del valor crítico, provoca falta de homogeneidad en la superficie de las piezas, alterando de esta manera el perfil de difusión a lo largo de la capa carburizada. Caso contrario ocurrió al trabajar con un exceso de caudal como en el caso de la muestra M3; donde la formación y deposición de hollín en el interior del reactor se esperaba, por las altas concentraciones de carbono que se manejan. El flujo efectivo de carbono depende también de la superficie de carga, cuando la superficie es inferior a la superficie crítica, se constata que el flujo es constante, lo que genera un perfil homogéneo de carbono en la capa carburada, pero cuando se alcanza el valor de superficie crítica, el flujo observado disminuye dando como resultado un perfil de carbono desviado de lo teórico [7].

Las micrografías de la capa carburizada que se observan en las Figuras $5 \mathrm{~b}, 5 \mathrm{~d}$ y $5 \mathrm{f}$ corresponden con las muestras M1, M2 y M3 respectivamente. La muestra M1, figura 5b, corresponde a una microestructura en la capa carburada compuesta por una granos de ferrita (área clara) y perlita laminar (área oscura) característi$\cos$ de un acero hipoeutectoide. La profundidad de la capa carburada fue de $150 \mu \mathrm{m}$. La capa carburada de la muestra M2, figura 5d, corresponde una estructura eutectoide de perlita laminar, con una profundidad promedio de capa carburada de $350 \mu \mathrm{m}$, es de resaltar la presencia de grandes regiones de martensita (área clara) próximo a la superficie de la muestra; la presencia de elementos aleantes como el $\mathrm{Cr}$, Ni y Mo aumentan la templabilidad del acero AISI/SAE 8620, y a pesar de que el enfriamiento posterior al ciclo de carburización fue realizado al aire, se lograron las condiciones para que en esta región de la muestra ocurra una transformación a martensita. Por último en la muestra M3, figura 5f, se aprecia una microestructura hipereutectoide de perlita laminar bordeada por una red de cementita y áreas de martensita próximo a la superficie de la capa carburada, de forma análoga a la muestra M2. Además la profundidad promedio de la capa carburizada fue de $450 \mu \mathrm{m}$.

En la figura 5a, 5c y 5e se muestra el perfil de microdureza a lo largo de la capa carburizada para los tratamientos M1, M2 y M3, respectivamente, en función de la microestructura obtenida. Para la muestra M1 se observa que el valor máximo de microdureza es de $250 \mathrm{HV}$, característico de una microestructura ferritoperlítica con $0.5 \%$ de carbono en superficie, para la muestra M2, la microdureza es de 400HV. Este incremento con respecto a M1, se atribuye al incremento de carbono en la superficie, el cual es de $0.8 \%$, además de la presencia de martensita en la capa carburizada. Por último para la muestra M3 se alcanza una microdureza de $450 \mathrm{HV}$, valor superior a M2 esencialmente debido al incremento en el contenido de carbono, el cual es de $1.1 \%$. Estos resultados concuerdan con lo presentado en la figura 6, en la cual se muestran los efectos comparativos de la variación de la dureza con el contenido de carbono y la microestructura desarrollada de acuerdo al proceso de enfriamiento de las muestras M1, M2 y M3. La máxima dureza se consigue con una microestructura totalmente martensítica con respecto a las durezas de las microestructuras ferrita-perlita o esferoidizadas en todo el rango de carbono.

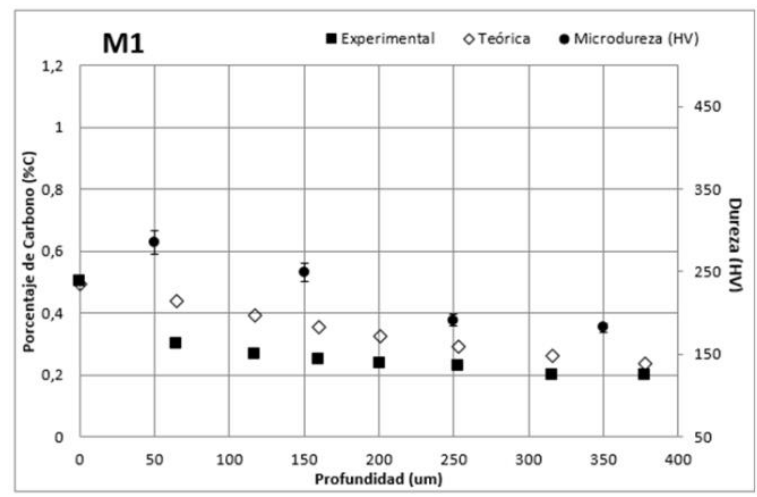

(a)

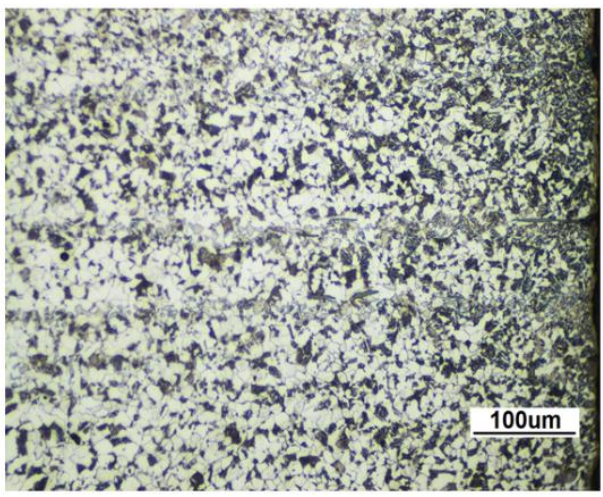

(b) 


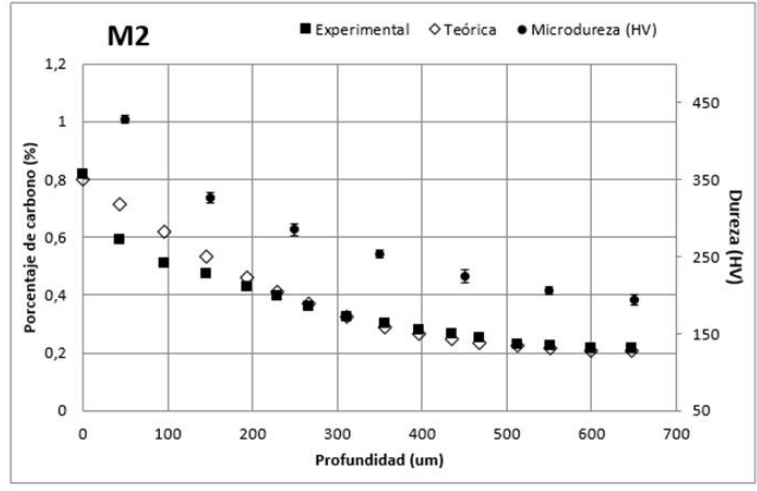

(c)

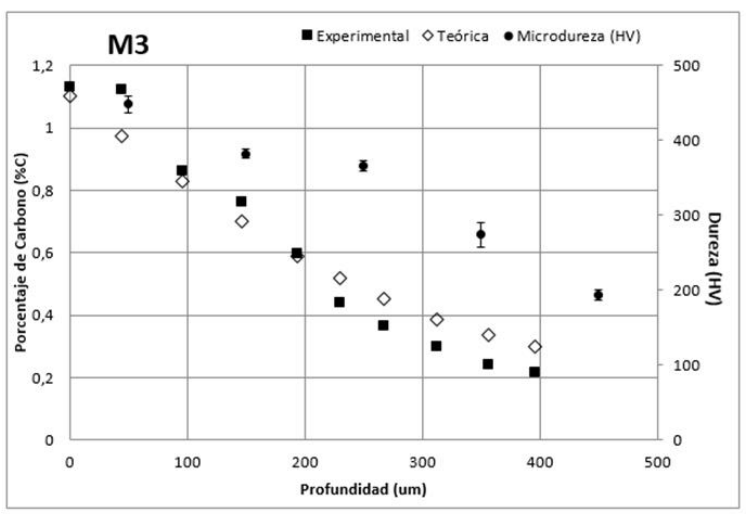

(e)

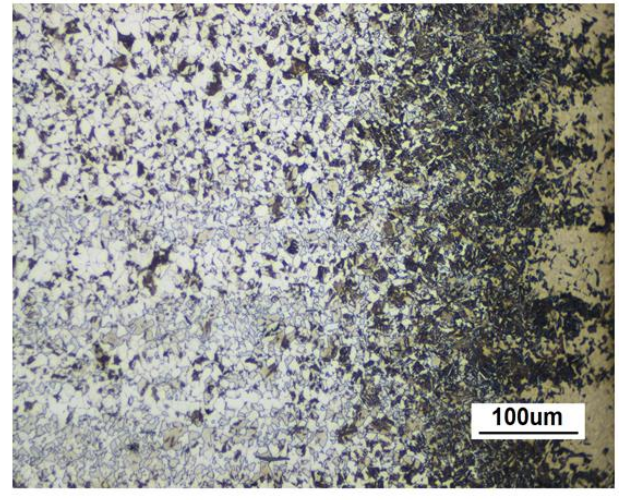

(d)

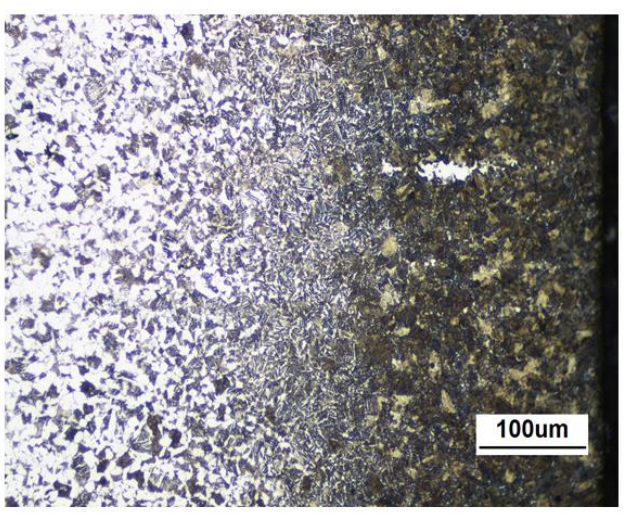

(f)

Figura 5: Perfiles de carbono, microdureza Vickers y micrografías ópticas para los niveles trabajados: (a-b) M1, (c-d) M2 y (e-f) M3.

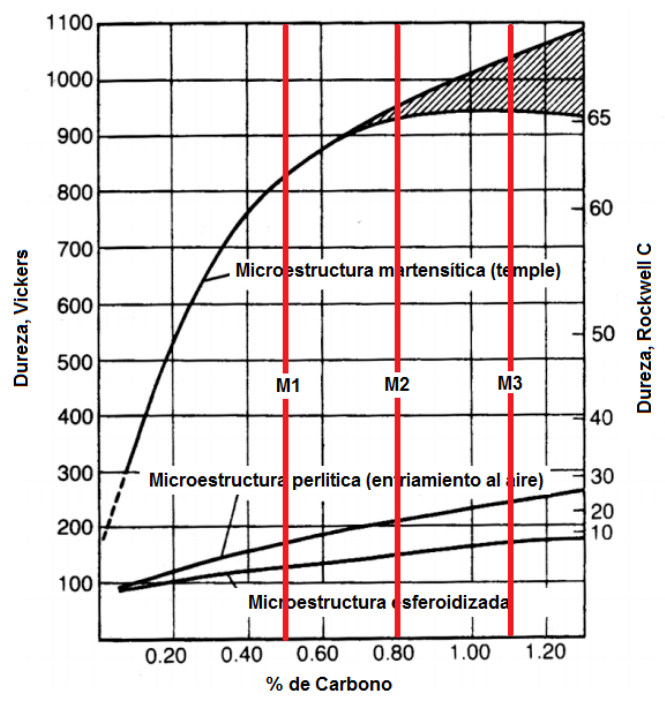

Figura 6: Dureza en función del contenido de carbono para distintas microestructuras [17].

\section{CONCLUSIONES}

La introducción de un flujo de acetileno del 30\%, 40\% y 45\% respecto al caudal total resultó en la obtención de porcentajes de carbono del $0.5 \%$ al $1.1 \%$, con profundidades de capa carburizada crecientes, y valores mayores de microdureza. Además se constató que a mayor flujo de acetileno se favorecen las reacciones de 
aromatización produciendo hollín.

Se verificó para las condiciones ensayadas que el acetileno tiene un proceso de descomposición completo en carbono naciente e hidrógeno gaseoso en la superficie del metal, logrando que el proceso de carburización sea eficaz.

\section{AGRADECIMIENTOS}

Al CODI de la Universidad de Antioquia por la financiación a través del proyecto PRG14-1-07, de la estrategia de sostenibilidad 2014-2015 y de la beca joven investigador CODI y a Colciencias por la beca Joven Investigador e Innovador 2014.

\section{BIBLIOGRAFÍA}

[1] VALENCIA, A., Tecnologia del tratamiento térmico de los metales. Editorail Universidad de Antioquia Medellin, 2009.

[2] HERRING, D. H., BREUER, D. J., LINDELL, G. D. "Selecting the Best Carburizing Method for the Heat Treatment of Gears", Disponível em: http://www.heat-treatdoctor.com/documents/Best $\% 20$ Method\%20for\%20the\%20Heat\%20Treatment\%20of\%20Gears.pdf

[3] KHAN, R. U., BAJOHR, S., GRAF, F. "Modeling of acetylene pyrolysis under steel vacuum carburizing conditions in a tubular flow reactor", Molecules, vol. 12, no. 3, pp. 290-296, 2007.

[4] KASPERSMA, J., PEARTREE, R. "Process for carburizing ferrous metals", Patente: CA1140438 A, Oficina de propiedad intelectual de Canadá, 1981.

[5] KARABELCHTCHIKOVA, O. Fundamentals of Mass Transfer in Gas Carburizing, Tesis de D.Sc., Worcester Polytechnic Instituite, 2007.

[6] HERRING, D. "Atmosphere gas carburizing - Case Studies, Lessons learned”, Ind. Heat., vol. 80, no. 10, pp. 16-18, 2012.

[7] CASTELAO, J. Tratamiento de cementación en hornos de baja presión, Tesis de D.Sc., Universidad Autonoma de Barcelona, 2014.

[8] HERRING, D., PETERS, R. "New-Formula Acetylene Cool for Heat Treatment", Gear Technol., no. 3, pp. 90-95, 2013.

[9] NORINAGA, K., DEUTSCHMANN, O. "Detailed Kinetic Modeling of Gas-Phase Reactions in the Chemical Vapor Deposition of Carbon from Light Hydrocarbons", Ind. Eng. Chem. Res., vol. 46, no. 11, pp. 3547-3557, 2007.

[10] WANG, Y., YANG, Z., ZHANG, F., et al., "Microstructures and mechanical properties of surface and center of carburizing $23 \mathrm{Cr}_{2} \mathrm{Ni}_{2} \mathrm{SiMo}$ steel subjected to low-temperature austempering", Mater. Sci. Eng. A, vol. 670, pp. 166-177, Jul. 2016.

[11] HERRING, D. H. "A Case for Acetylene Based Low Pressure Carburizing of Gears", Therm. Process., no. 3, pp. 40-45, 2012.

[12] FILLARI, G., MURPHY, T., GABRIELOV, I. "Effect of Case Carburizing on Mechanical Properties And Fatigue Endurance Limits of P/M Steels", Hoeganaes Corporation - Borg Warner Automotive, Livonia, MI 48150.

[13] KHAN, R. U., BAJOHR, S., BUCHHOLZ, D., et al., "Pyrolysis of propane under vacuum carburizing conditions: An experimental and modeling study", J. Anal. Appl. Pyrolysis, vol. 81, no. 2, pp. 148-156, 2008.

[14] KOROUŠIČ, B., LJUBLJANA, I. M. T. "Predicting of Reactions During Carburization and Decarburization of Steels in Controlled Atmospheres", Kovine, zlitine, Tehnol., vol. 30, no. 6, pp. 521-526, 1996.

[15] ASTM INTERNATIONAL, "E92-82: Standard Test Method for Vickers Hardness of Metallic Materials", ASTM Stand., vol. 82, no. Reapproved 2003, pp. 1-9, 2000.

[16] ASTM INTERNATIONAL, "E3-11: Standard Guide for Preparation of Metallographic Specimens 1", ASTM Int., vol. i, pp. 1-12, 2011.

[17] UNIVERSIDAD NACIONAL DE LA PLATA, "Dureza y Templabilidad en aceros", Estructura y propiedades de las aleaciones, pp. 1-21. 2009 